\title{
Utilization of Classical Scaling Technique in Sustaining Fault Detection Performance in Process Monitoring
}

\author{
Mohd Yusri Mohd Yunus ${ }^{1,}$, , Jie Zhang' ${ }^{2}$, Sajjad K. Al-Amshawee ${ }^{1}$ \\ ${ }^{1}$ Faculty of Chemical and Process Engineering Technology, Universiti Malaysia Pahang, 26300 Gambang, Pahang, Malaysia \\ ${ }^{2}$ School of Chemical Engineering and Advanced Material, Newcastle University, Newcastle Upon Tyne, NE1 7RU, UK
}

\begin{abstract}
Multivariate Statistical Process Monitoring (MSPM) fundamentally adopts the conventional Principal Component Analysis (CPCA) as the main platform for data compression. The main challenge though, the association nature of most industrial process variables are highly non-linear. As a result, the risks of applying the conventional approach of MSPM within this context may include sluggish or failed in detection, misinterpretation of signals, incorrect fault diagnosis and also inflexible as well as insensitive to changing of operating modes. In addressing the issue, this paper introduces new sets of monitoring parameters i.e. Sm2, Sr2 and Sr3, which have been derived within the frameworks of Classical Scaling (CMDS) and Procusters Analysis (PA) methods. The overall fault detection performance that applied based on the Tennessee Eastman Process (TEP) cases show that the Sr3 can detect the faults particularly for abnormal events number 3, 9, 15 and 19 in higher rate compared to the cPCA-MSPM system. This proves that the new monitoring statistics work effectively in avoiding missed detection during monitoring which cannot be addressed effectively by the traditional monitoring system.
\end{abstract}

\section{KEYWORDS}

Multivariate statistical, process monitoring (MSPM), principal component analysis $(P C A)$, classical scaling (CMDS).

\section{INTRODUCTION}

In principle, Multivariate Statistical Process Monitoring (MSPM) is an established as well as comprehensive statistical-tool driven system which primarily functioned to detect and diagnose abnormalities during operation. Among of the initial frameworks have been proposed in a number of numerous studies which focusing more on chemical-based industry (Kourti \& MacGregor, 1995; J. F. MacGregor \& Kourti, 1995; Martin et al., 1996). Two main statistics, namely Hotelling's $T^{2}$ and Squared Prediction Errors (SPE), have been extensively applied particularly for fault detection operation. By conception, the first is highly regarded to the magnitude of centrality, whereas the second critically analyses the stability of process variable correlations under investigation. Both parameters are typically ran together and complementary during monitoring implementation. Technically, a fault will be signalled if any one of these statistics located beyond of the monitoring limits (normally set at $99 \%$ confidence level) consecutively on the multivariate Shewhart's control chart.

In general, MSPM typically utilizes the conventional Principal Component Analysis (cPCA) approach as its foundation for data compression. This procedure is very important particularly to simplify the monitoring operation by transforming all the monitored variables linearly into multivariate numerical, or popularly known as PC scores (which lesser in numbers compared to the original variables) prior charting the monitoring statistics. However, various process industries suffer from acute non-linear behaviour. As a result, the risks of applying the conventional approach of MSPM within this context may include sluggish or failed in detection, misinterpretation of signals, incorrect fault diagnosis and also inflexible as well as insensitive to changing of operating modes. In addressing these issues, (Yunus, 2012) has proposed Classical Scaling (CMDS), in which, the technique works almost identical to that of cPCA, but fundamentally different by means of variable correlations. In (Yunus, 2012), the author has also explained the benefits as well as demonstrated the unique features of CMDS against cPCA on a simulated process of continuous stirred tank reactor (CSTR) with recycle system. However, the monitoring statistics proposed in (Yunus, 2012) suffer from high frequency of missed detection situations which are similar to cPCA. In other words, both cPCA and CMDS in (Yunus, 2012) are having difficulty to 'sustain' the detection signals if the process is highly non-linear. In particular, this study defines the term 'sustain' corresponding to the ability of the monitoring system detecting faults consistently in the abnormal region of the control chart without allowing the statistics moving back to the normal area as long as the root cause that pertaining to the signals are retained in the process operation. In this light, a newly monitoring statistic which has been derived as well as expanded from (Yunus, 2012) is proposed in addressing the issue. Hence, the objective of this study is to investigate how reliable of the new monitoring statistic can sustain the detection signal of fault operation in oppose to cPCA based on the specified case studies. 


\section{MULTIVARIATE STATISTICAL PROCESS MONITORING SYSTEM}

\section{Conventional MSPM System \& Its Advanced Applications}

MSPM basically applies linear-based foundation to correlate all the monitored variables in the form of matrices. The typical representations of this association either by means of variance-covariance or correlation matrix structure are shown in (1) and (2), respectively.

$$
\begin{aligned}
\text { Variance-covariance matrix, } \mathbf{C}=\left[\begin{array}{cccc}
c_{1,1} & c_{1,2} & \ldots & c_{1, m} \\
c_{2,1} & c_{2,2} & \ldots & c_{2, m} \\
\cdot & \cdot & \cdot & \cdot \\
\cdot & \cdot & \cdot & \cdot \\
\cdot & \cdot & \cdot & \cdot \\
c_{m, 1} & c_{m, 2} & \ldots & c_{m, m}
\end{array}\right] \\
{\left[\begin{array}{cccc}
r_{1,1} & r_{1,2} & \ldots & r_{1, m} \\
r_{2,1} & r_{2,2} & \ldots & r_{2, m} \\
\cdot & \cdot & \cdot & \cdot \\
\cdot & & & \cdot \\
\cdot & & & \cdot \\
r_{m, 1} & r_{m, 2} & \cdots & r_{m, m}
\end{array}\right] }
\end{aligned}
$$

where sample variances or covariances (var-cov), $c_{a b}$, (in which ' $a$ ' equals to $1,2, \ldots$ ' $m$ ' while ' $b$ ' is referring to $1,2, \ldots m$ ), and also correlation measure, $r_{a b}$ (in which $a=1,2, \ldots$ ' $m$ ' while $b=1,2, \ldots m$ ) are given by (3) and (4), respectively:

$$
\begin{gathered}
c_{a b}=\frac{1}{(n-1)} \sum_{i=1}^{n}\left(\mathbf{x}_{i a}-\overline{\mathbf{x}}\right)^{T}\left(\mathbf{x}_{i b}-\overline{\mathbf{x}}\right) \\
r_{a, b}=\frac{c_{a, b}}{\sqrt{c_{a, a}} \sqrt{c_{b, b}}}
\end{gathered}
$$

where $\mathbf{x}$ is a particular column (vector) of the original matrix data, $\mathbf{X}_{n \times m}$ ( $n$ and $m$ are number of samples and variables, respectively). The PCs scores are obtained, as shown in (5) and (6), simply by the product of the original multivariate data (which usually normalized), $\mathbf{X}$, and eigenvectors that extracted from the var-cov or correlation matrix (Everitt \& Jackson, 1992). The complete procedures of connecting the PC scores and monitoring statistics can be obtained in (Kourti \& MacGregor, 1995; J. F. MacGregor \& Kourti, 1995; Martin et al., 1996).

$$
\begin{gathered}
\mathbf{P}_{n \times m}=\mathbf{X}_{n \times m} \mathbf{V}_{m \times m} \\
\mathbf{P}=\left[\begin{array}{ccc}
x_{1,1} v_{1,1}+x_{1,2} v_{2,1}+\cdots+x_{1, m} v_{m, 1} & \ldots & x_{1,1} v_{1, m}+x_{1,2} v_{2, m}+\cdots+x_{1, m} v_{m, m} \\
x_{2,1} v_{1,1}+x_{2,2} v_{2,1}+\cdots+x_{2, m} v_{m, 1} & \ldots & x_{2,1} v_{1, m}+x_{2,2} v_{2, m}+\cdots+x_{2, m} v_{m, m} \\
\vdots & \vdots & \vdots \\
x_{n, 1} v_{1,1}+x_{n, 2} v_{2,1}+\cdots+x_{n, m} v_{m, 1} & \ldots & x_{n, 1} v_{1, m}+x_{n, 2} v_{2, m}+\cdots+x_{n, m} v_{m, m}
\end{array}\right]
\end{gathered}
$$

Procedures that highlighted in (1) to (6) are the core of cPCA. There are also other applications of multivariate reduction techniques which have been utilised for monitoring including Partial Least Square (Nomikos \& MacGregor, 1995), Independent Component Analysis (Lee et al., 2004), Factor Analysis (Amigo et al., 2008) and Partial Correlation Analysis (Ibrahim, 1997). However, all of these extensions perceive the variable correlations as similar to the concept of cPCA, and thus, the earlier issues remained valid. Meanwhile, most advanced studies of MSPM have been on the improvement of cPCA itself which consists of dynamic PCA (Ku et al., 1995), Multi-scale PCA (Bakshi, 1998), Multiblock PCA (John F. MacGregor et al., 1994) and also Non-linear PCA (Zhang et al., 1997). Among of these approaches, 
Non-linear PCA is the only mechanism which specifically highlights and radically proposed modification on the traditional linear-based model structure by implementing principal curve technique. Alternatively, (Yunus, 2012) has proposed CMDS technique for monitoring application, which is also introducing a new scheme for constructing the variable model. Critical comments on the principal curve approach can be also found in (Yunus, 2012).

\section{Classical scaling and process monitoring}

Multidimensional Scaling (MDS) generally is an explorative technique, which initially as well as popularly adopted by the social science area (Oppenheim \& Torgerson, 1961), particularly to provide the sense of general structure by means of graphical representation of any large scale data that investigated (Kruskal, J.B., and Wish, 1978). One of the core elements is dissimilarity/similarity measure which conceptually applied to represent the association among of the objects (samples) such that higher degree of connection between two objects is depicted by having both points located closely together relative to the other pair of settings which loosely connected (Borg \& Groenen, 1997; T. F. Cox, 2005; T. F. Cox \& Cox, 1994; Takane, 2004). In the cPCA term, this higher degree of connection can be in the range of 0.7 to 1.0 on the correlation matrix scale. In (T. F. Cox \& Cox, 1994), the authors have suggested various options in representing the distance of the quantitative data which consists of Euclidean, City-block, Mahalanobis and many others.

According to (Oppenheim \& Torgerson, 1961), CMDS is originated from a study that published in (Trevor F. Cox, 2001). The term 'metric' is also synonymous with CMDS because it assumes the input data to be in the form of ratioscaled distance (T. F. Cox, 2003). Generally, there are four basic steps considered in developing the CMDS scores (Borg \& Groenen, 1997; T. F. Cox \& Cox, 1994):

i. Firstly, the squared dissimilarity matrix, $\Delta^{2}$, is computed.

ii. Then, the double-centring operation is applied on matrix $\Delta^{2}$ to obtain $\mathbf{B}_{\Delta}$.

iii. Next, the eigen decomposition of $\mathbf{B}_{\Delta}$ is calculated.

iv. Finally, the Cartesian coordinate matrix, $\mathbf{X}_{E}$, is found by multiplication of positive eigenvalues with their corresponding eigenvectors.

For instance, the following illustrates the technical procedures in developing the multivariate scores (PCs scores) using the CMDS technique. Suppose the Euclidean Scale is chosen to be applied, and hence, the squared inter-distances (dissimilarity) matrix among of the objects (samples) can be calculated via (7).

$$
\Delta^{2}=D^{2}=c 1^{\prime}+1 c^{\prime}-2 X X^{T}
$$

where $\mathbf{D}^{\mathbf{2}}$ is the squared dissimilarity matrix in terms of Euclidean distance, $\mathbf{1}$ is a vector containing element of 1 with size of column in $\mathbf{X}, \mathbf{c}$ is a vector containing the diagonal elements of $\mathbf{X X}^{\mathbf{T}}$, and is the Euclidean's major product moment. Then, the final form of $\boldsymbol{\Delta}^{\mathbf{2}}$ is given by way of (8):

$$
\boldsymbol{\Delta}^{\mathbf{2}}=\mathbf{D}^{\mathbf{2}}=\left[\begin{array}{cccc}
D_{11}^{2} & D_{12}^{2} & \ldots & D_{1 n}^{2} \\
D_{21}^{2} & D_{22}^{2} & \ldots & D_{2 n}^{2} \\
\vdots & \vdots & \ddots & \vdots \\
D_{n 1}^{2} & D_{n 2}^{2} & \ldots & D_{n n}^{2}
\end{array}\right]
$$

Next, by applying the double centring operation on $\Delta^{\mathbf{2}}$, a corrected scalar product matrix is generated, which means the data origin are re-located automatically to be on the centre (or centroid) of the data as shown as follows:

$$
\begin{aligned}
& \text { Corrected scalar product matrix }=\mathbf{B}_{\Delta}=-\frac{1}{2} \mathbf{J}_{\mathbf{n}} \Delta^{2} \mathbf{J}_{\mathbf{n}} \\
& \text { where, } \mathbf{J}_{n}=\text { double centring }=\left(\mathbf{I}_{n}-\mathbf{1}_{n} \mathbf{1}_{n}^{\prime} / n\right) \\
& \text { Therefore, } \quad \mathbf{B}_{\Delta}=-\frac{1}{2} \mathbf{J}\left(\mathbf{c} \mathbf{1}^{\prime}+\mathbf{1} \mathbf{c}^{\prime}-2 \mathbf{X X} \mathbf{X}^{T}\right) \mathbf{J} \\
& =-\frac{1}{2} \mathbf{J c 1} \mathbf{J}-\frac{1}{2} \mathbf{J} 1 \mathbf{c}^{\prime} \mathbf{J}+\frac{1}{2} \mathbf{J}\left(2 \mathbf{B}_{E}\right) \mathbf{J} \\
& \text { As } \mathbf{1}^{\prime} \mathbf{J}=\mathbf{J} \mathbf{1}=\mathbf{0} \text {, and thus: } \mathbf{B}_{\Delta}=-\frac{\mathbf{1}}{\mathbf{2}} \mathbf{J c 0}-\frac{1}{\mathbf{2}} \mathbf{0} \mathbf{c}^{\prime} \mathbf{J}+\mathbf{J B J} \\
& =\mathbf{J B J}(\text { corrected scalar product matrix })
\end{aligned}
$$

By this, $\mathbf{B}_{\Delta}$ is a symmetrical and positive semi-definite matrix. Later, the eigen decomposition of $\mathbf{B}_{\Delta}$ is calculated by (Oppenheim \& Torgerson, 1961):

$$
\mathbf{B}_{\Delta}=\mathbf{U D U}^{\mathrm{T}}
$$

where $\mathbf{U}$ is an orthonormal matrix of eigenvectors and $\mathbf{D}$ is a diagonal matrix of eigenvalues with customary order such that $\lambda_{1} \geq \lambda_{2} \geq \cdots \geq \lambda_{n}$. At the final step, the recovery coordinate, usually by means of Euclidean space, $\mathbf{X}_{E}$, is simply determined by:

$$
\mathbf{X}_{E}=\mathbf{U}_{+} \mathbf{D}_{+}^{0.5}
$$


where $\mathbf{D}_{+}^{\mathbf{0 . 5}}$ is a diagonal matrix with all elements of $\lambda_{i}^{0.5}$ that are greater than zero and $\mathbf{U}_{+}$is the corresponding eigenvectors of those selected $\lambda_{i}^{0.5}$.

In addition, CMDS minimizes the loss function of scalar product or namely Strain, $S$, as shown in (16). Meanwhile, it can be also alternatively defined as the comparison between the total variance in the original scalar product matrix, $\mathbf{B}_{\Delta}$, with the total variance of a derived scalar product matrix from the new configuration of Euclidean space coordinate, $\mathbf{B}_{\boldsymbol{E}}$ (Oppenheim \& Torgerson, 1961).

$$
S=\Sigma\left|\mathbf{B}_{E}-\mathbf{B}_{\Delta}\right|^{2}
$$

A comprehensive revision on the application of MDS in monitoring has been reported in (Yunus, 2012). In summary, the original idea was proposed in (Trevor F. Cox, 2001), and also described in (T. F. Cox, 2003). There were two approaches have been developed. In particular, the first proposed the multivariate scores in terms of sample configuration, whereas the second adopted the variable profile scores. Hence, whenever a fault happened, the corresponding samples or variables will be moving away from the normal cluster either in a great magnitude of gradually. However, (Yunus, 2012) perceives the variable profile as rather advantageous as well as informative compared to the conventional score configuration for it shows directly which variables are responding to the situation that connected. However, neither in (Trevor F. Cox, 2001) nor (T. F. Cox, 2003) specifically suggested any mechanism in associating the CMDS scores with any established statistics for monitoring.

As a result, three general CMDS monitoring frameworks have been proposed in (Yunus, 2012) particularly to extend the proposed methodology reported in (Trevor F. Cox, 2001). However, the proposed systems only utilise the multivariate variable score profiles for monitoring. The first framework, which adopts the moving window scheme, directly obtain the first monitoring statistic by way of measuring the resultant vector distance of the CMDS scores from the centre (which inspired by the original idea proposed in (Trevor F. Cox, 2001)). This parameter conceptually relates to the application of Hotelling's $T^{2}$ statistic in the conventional MSPM. Meanwhile, the second monitoring statistic is simply obtained by calculating the summation of squared errors in terms of the dissimilarity measure that applied (only Euclidean and Cityblock were analysed). This second parameter is however imitates the representation of SPE which specifically analysing the degree of association among of the variables that monitored. Nonetheless, this particular monitoring framework does not particularly introduce a mechanism of loading factor which typically used in the cPCA approach. As a result, two additional frameworks were proposed that particularly utilizing Procrusters Analysis (PA) in emulating the conceptual application of loading factors in cPCA. The main difference between the two methods however, the third system employs dynamic loading calculation in projecting the scores as oppose to the second approach. In analysing the whole CMDS monitoring frameworks against to that of cPCA performance, the second CMDS system (CMDS-PA) was found the most stable in terms of fault detection time particularly based on the case study that investigated.

\section{CMDS-PA MONITORING FRAMEWORKS}

The proposed procedures of CMDS-PA which have been upgraded from (Yunus, 2012) is shown in Figure 1. The first step basically involves collecting the normal operating condition (NOC) data, which is importantly applied in developing the correlation model for monitoring. In this study, the data is assumed to be pre-treated initially, whereby the comprehensive procedures of raw data pre-treatment can be referred in (Molina et al., 2011). The collected set of NOC data is then undergone normalization or standardized as according to zero mean and one standard deviation, by which all the variables are treated equally regardless of the scales of operational measurements. In step 2, the original set of NOC data is divided into two parts - $\mathbf{X}_{\mathbf{N O C} 1}$ and $\mathbf{X}_{\mathbf{N O C 2}}$. This is mainly important for developing the benchmarked multivariate model particularly using the first set, $\mathbf{X}_{\mathbf{N O C} 1}$, whereas the second, $\mathbf{X}_{\mathbf{N O C} 2}$, is utilised to obtain the progression statistic profile especially for calculating the monitoring limits. The typical CMDS procedures, as explained in (7) to (15) previously, are employed in converting both $\mathbf{X}_{\text {NOC1 }}$ and $\mathbf{X}_{\text {NOC2 }}$ into $\mathbf{Y}_{\text {NOC1 }}$ and $\mathbf{Y}_{\text {NOC2 }}$ respectively. Then, the PA procedures are applied as described in the following (Borg \& Groenen, 1997):

i. Computation of the minor product moment between the first NOC scores, $\mathbf{Y}_{\text {NOC1 }}$ (originated from $\mathbf{X}_{\text {NOC1) }}$ ) and the second NOC scores, $\mathbf{Y}_{\text {NOC2 }}$ (originated from $\mathbf{X}_{\mathrm{NOC} 2}$ ).

$$
\mathbf{C}_{\mathbf{P A}}=\mathbf{Y}_{\text {NOC1 }}{ }^{\mathrm{T}} \mathbf{J}_{\mathrm{m}} \mathbf{Y}_{\text {NOC2 }}
$$

where $\mathbf{J}_{\mathbf{m}}$ is given in (9) based on the size of variables instead of samples.

ii. Decomposition of $\mathbf{C}_{\mathbf{P A}}$ into the eigen basic structures.

$$
\mathbf{C}_{\mathbf{P A}}=\mathbf{P}_{\mathbf{P A}} \mathbf{V}_{\mathbf{P A}} \mathbf{P}_{\mathbf{P A}}{ }^{\mathrm{T}}
$$

where $\mathbf{P}_{\mathbf{P A}}$ and $\mathbf{V}_{\mathbf{P A}}$ are the eigenvectors and eigenvalues matrices of $\mathbf{C}_{\mathbf{P A}}$ respectively.

iii. Identification of the optimal rotation matrix:

$$
\mathbf{R}=\mathbf{P}_{\mathbf{P A}} \mathbf{P}_{\mathbf{P A}}{ }^{\mathrm{T}}
$$

iv. Identification of the optimal dilation scale:

$$
s=\left(\operatorname{tr} \mathbf{Y}_{\mathrm{NOC} 1}{ }^{\mathrm{T}} \mathbf{J}_{\mathrm{m}} \mathbf{Y}_{\mathrm{NOC} 2} \mathbf{R}\right) /\left(\operatorname{tr} \mathbf{Y}_{\mathrm{NOC} 2}{ }^{\mathrm{T}} \mathbf{J}_{\mathrm{m}} \mathbf{Y}_{\mathrm{NOC} 2}\right)
$$

v. Identification of the optimal translation vector:

$$
\mathbf{t}=\left(\mathbf{Y}_{\text {NOC1S }} \mathbf{Y}_{\text {NOC2 } 2} \mathbf{R}\right)^{\mathrm{T}} \mathbf{1} / m ; m=\text { no. of variables }
$$


vi. The reproduction of Moving Window Observation Samples of Normal Operating Condition (MWOS-NOC) scores projected by PA for sample ' $k$ ' is given by:

$$
\mathbf{Y}_{\mathbf{P A}-\mathbf{N O C}(k)}=S \text { YMWOS-NOC }(k) \mathbf{R}+\mathbf{1 t}^{\mathrm{T}}
$$

where $\mathbf{Y}_{\text {MwOS-NOC }(k)}=$ new CMDS scores of MWOS-NOC samples at time

' $k$ ' that converted from $\mathbf{X}_{\text {MwOS-NOC }(k)}$ based on the moving window scheme.

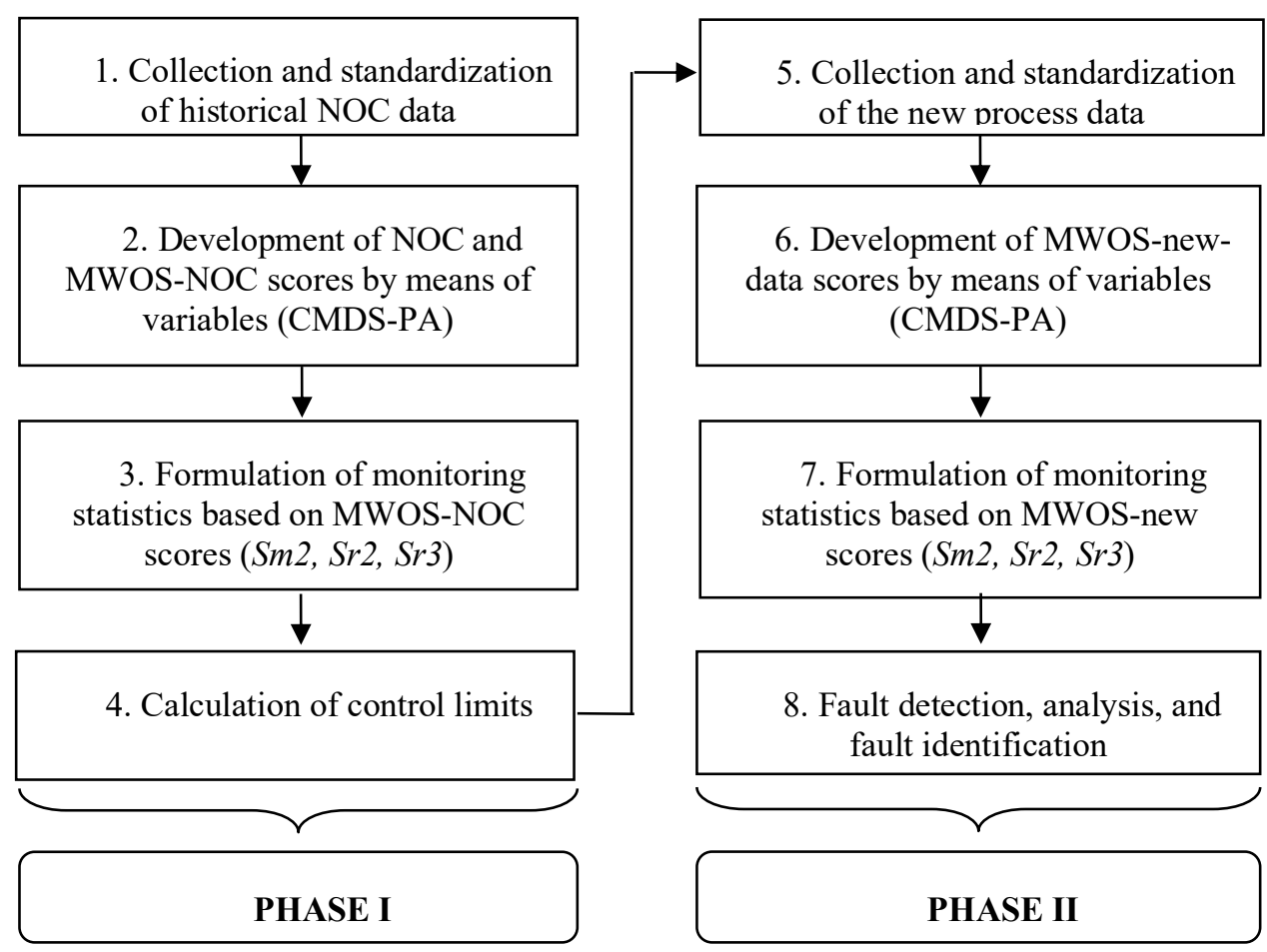

Figure 1. CMDS-PA based MSPM framework

In step 3, three monitoring statistics are calculated in representing the monitoring progression. The first statistic, which denoted in (23), is the sum of squared errors in terms of projected variable vectors in the reduced dimensional space between YPA and YPA-NOC1 configurations. This particular parameter is conceptually addressed the magnitude of deviation of current process progression from the centre (further explanation can be found in (Yunus, 2012)).

$$
S_{m 2}=\sum_{i=1}^{m}\left(\sum_{j=1}^{p}\left[y_{P A-N O C(k)}(i, j)-y_{P A-N O C 1}(i, j)\right]^{2}\right)
$$

where $y_{P A-N O C(k)}$ and $y_{P A-N O C 1}$ are a particular score coordinate in YPA-NOC(k) and YPA-NOC1 by which can be calculated as in (24) and (25) respectively. The term ' $\mathrm{m}$ ' is referring to the number of variables, ' $p$ ' corresponds to the number of dimensions that compressed, and ' $\mathrm{k}$ ' is a particular moving window observation (MWOS) timing.

$$
\mathbf{Y}_{\mathrm{PA}-\mathrm{NOC} 1}=S \mathbf{Y}_{\mathrm{NOC} 2} \mathbf{R}+1 \mathbf{t}^{\mathrm{T}}
$$

Meanwhile, the second statistic regards to measuring the correlation consistency as illustrated in (23). From (23), technically this parameter measures the sum of squared errors in terms of dissimilarity measures between the $\mathbf{Y}_{\mathbf{P A}-\mathrm{NOC}(k)}$ and Y YA-NOC1 configurations. Detail descriptions are given in (Yunus, 2012).

$$
S_{r 2}=\sum\left(\sum_{i=1}^{m} \sum_{j=1}^{m}\left[d_{P A-N O C(i j)}-\delta_{P A-N O C 1(i j)}\right]^{2}\right) ; i \neq j
$$

where $d_{P A-N O C(k)}$ and $d_{P A-N O C 1}$ are a particular inter-distance measure in YPA-NOC(k) and YPA-NOC1 respectively. The third statistic, $\mathrm{Sr} 3$ is the newly monitoring parameter that proposed through this particular study and expressed mathematically as in (26), whereby the terms are similar to that of $S_{r 2}$. This particular parameter is proposed to complement the performance of $S_{r 2}$ because $S_{r 2}$ is sometimes found to be problematic during the initial stage of transition 
particularly when transforming from the normal to abnormal states. As a result, $S_{r 2}$ tends to be sluggish to detect fault compared to $S_{m 2}$.

$$
S_{r 3}=\sum_{i=1}^{m} \sum_{j=1}^{m} \frac{d_{P A-N O C(k i j)}}{\delta_{P A-N O C 1(i j)}} ; i \neq j \text { at time } k
$$

The last task of phase 1, which is step 4 deals specifically in developing the monitoring limits. As suggested in (Yunus, 2012), this study adopts the chi-squared distribution as shown in (27).

$$
\lim _{\alpha}=(v / 2 \bar{m}) \chi_{2 m^{2} / v, \alpha}^{2}
$$

where $\alpha$ equals to 0.05 and 0.01 for warning (95\%) and control (99\%) limits respectively, $\bar{m}$ and $v$ are respectively representing the means and variances for each of the statistics. The phase II procedures, particularly steps 5 until 7 , basically follow the same order as in phase I. In particular, the new samples are standardized as according the means as well as standard deviations that obtained in Phase I. Then, the scores are simply obtained through the CMDS normal procedures, as shown in (7) to (15), whereby the original new samples are dynamically changed through moving window mechanism ( $\left.\mathbf{X}_{\text {Mwos-new }(k)}\right)$ in order to compute the new sample CMDS scores ( $\left.\mathbf{Y}_{\mathbf{M w O S} \text {-new }(k)}\right)$. The projecting algorithm for the new sample scores is shown in (28).

$$
\mathbf{Y}_{\mathbf{P A - n e w}(k)}=S \mathbf{Y}_{\mathbf{M w O S}-\mathrm{new}(k)} \mathbf{R}+\mathbf{1 t}^{\mathrm{T}}
$$

where $\mathbf{Y}_{\text {PA-new }(k)}=$ reproduction of the scores projected by PA at MWOS-new sample ' $k$ ', whereas $\mathbf{Y}_{\text {Mwos-new }(k)}=$ multivariate scores of MWOS sample ' $k$ ' developed by CMDS. This procedure is then completed by calculating the three monitoring statistics as shown in (23), (24) and (25) respectively. In step 8, a fault is detected whenever at least five (5) statistics (any of the proposed parameters) located consecutively beyond the $99 \%$ monitoring limits on the control charts.

\section{RESULTS AND DISCUSSION}

This study has applied Tennessee Eastman Process (TEP) (Downs \& Vogel, 1993) case study in evaluating the strength of the proposed systems. From the literature, this particular process has been widely investigated in analysing various applications of new control strategies (Molina et al., 2011; Zheng, 1998) as well as monitoring systems (Juricek et al., 2000). TEP comprises of five major unit operations including an exothermic two-phase reactor, a vapor-liquid separator, a product condenser, a product stripper and a recycle compressor. It mainly produces two major products with one material as side product particularly by using four main reactants. There are 52 variables are utilised for monitoring, in which it consists of two main groups - 41 measured variables and 11 manipulated variables (the details can be obtained in (Chiang et al., 2000)). In (Chiang et al., 2000), there are twenty different types of malfunction operations are proposed as listed in Table 1.

Table 1. Process Faults for Tennessee Eastman Process

\begin{tabular}{clll}
\hline Fault No. & Description & $\begin{array}{l}\text { Fault } \\
\text { No. }\end{array}$ & Description \\
\hline 1 & $\begin{array}{l}\text { A/C feed ratio, B composition constant } \\
\text { (stream 4) }\end{array}$ & 11 & Reactor cooling water inlet temperature \\
2 & B composition, A/C ratio constant (stream 4) & 12 & Condenser cooling water inlet temperature \\
3 & D feed temperature (stream 2) & 13 & Reaction kinetics \\
4 & Reactor cooling water inlet temperature & 14 & Reactor cooling water valve \\
5 & Condenser cooling water inlet temperature & 15 & Condenser cooling water valve \\
6 & A feed loss (stream 1) & 16 & Unknown \\
7 & C header pressure loss-reduced availability & 17 & Unknown \\
8 & (stream 4) & 18 & Unknown \\
9 & A,B,C feed composition (stream 4) & 19 & Unknown \\
10 & D feed temperature (stream 2) & 20 & Unknown \\
\hline
\end{tabular}

This study has investigated simulation data that implemented in [29]. The number of samples of the NOC training data NOC is 500; meanwhile the number of samples of testing data, which includes NOC and fault data sets is 960 individually. In this study, the fault signal has been introduced at the sampling time of 160 for each of the process faults respectively. Initially, the complete procedures of conventional MSPM were implemented and the Fault Delayed Detection Time (FDDT) were observed for each of the cases that investigated. This is followed by the application of 
CMDS-PA monitoring algorithms on each of the 20 cases respectively whereby the FDDT were also collected. Lastly, the results in terms of FDDT on those TEP cases were obtained individually by using the integrated framework of CMDSPA system. All applications have been tested based two main settings - 20 and 40 PCs/dimensions, whereby the 20 and 40 dimensions relate to almost $70 \%$ and $90 \%$ of data variation, respectively. In addition, the window size settings of 10 , 20 and 30 were also analysed in each of the dimension applications particularly for CMDS-PA and CMDS-PA-PCA systems. This study also has utilised the Euclidean distance as the main dissimilarity scale in constructing the overall variable relationship structure, whereby this particular scale has been identified as the most reliable performance compared to other measures that available for monitoring (Yunus, 2012).

\section{Phase I monitoring results}

The overall results of False Alarm Rate (FAR) analysis of CMDS-PA system are shown in Table 2 (the FAR results for the cPCA system are zero for all applications, and thus, not presented in this particular table). FAR is calculated based on the total number of samples which located beyond the $99 \%$ limit over total number of training and testing samples respectively. In general, the overall results of Table 2 depicts that the rates are generally lower than $10 \%$ which corresponding to testing data set. This suggests that the proposed monitoring limits should be robust in differentiating the normal and abnormal trending. It also indicates that if there are false alarm events take place, then the situation should be temporary within short period of time.

Table 2. The Summary of FAR Analysis Results based on CMDS-PA System

\begin{tabular}{|c|c|c|c|c|c|c|c|c|c|}
\hline \multirow{2}{*}{\multicolumn{10}{|c|}{$\begin{array}{c}\text { FALSE ALARMS RATE (\%) } \\
\text { NOC 500 / Dim 20 / Euclidean Distance }\end{array}$}} \\
\hline & & & & & & & & & \\
\hline \multirow[b]{2}{*}{ Data Set } & \multicolumn{3}{|c|}{ Win10 } & \multicolumn{3}{|c|}{ Win 20} & \multicolumn{3}{|c|}{ Win 30} \\
\hline & $\cong$ & $\tilde{\bar{\omega}}$ & $\tilde{n}$ & $\cong$ & $\tilde{\bar{\omega}}$ & $\stackrel{n}{\hbar}$ & ఓ & $\tilde{i}$ & $\tilde{s}$ \\
\hline Training & - & 0 & o & - & N & o & 0 & m & o \\
\hline Testing & m & 0 & o & $\checkmark$ & o & o & o & o & 0 \\
\hline \multicolumn{10}{|c|}{ NOC 500 / Dim 40 / Euclidean Distance } \\
\hline \multirow[b]{2}{*}{ Data Set } & \multicolumn{3}{|c|}{ Win10 } & \multicolumn{3}{|c|}{ Win 20} & \multicolumn{3}{|c|}{ Win 30} \\
\hline & $\cong$ & $\stackrel{\sim}{\tilde{\omega}}$ & $\tilde{\hbar}$ & $\cong$ & $\tilde{\bar{\omega}}$ & $\tilde{n}$ & $\cong$ & $\tilde{\omega}$ & $\stackrel{n}{n}$ \\
\hline Training & - & 0 & o & - & $N$ & 0 & 0 & $m$ & o \\
\hline Testing & $m$ & 0 & o & $\sim$ & o & 0 & 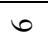 & o & 0 \\
\hline
\end{tabular}

\section{Phase II Monitoring Results}

The overall FDDT of CMDS and cPCA systems particularly for the 20 and 40 dimensions/PCs settings respectively are shown in Table 3. From Table 3, the results reveal that the CMDS-PA system performed comparatively superior relative to cPCA because the NFD achievements are higher than that of cPCA respectively at both dimension settings (65\% fastest detection which represented by the bolded numbers). In this regard, cPCA has shown $50 \%$ succession of fastest detections relative to CMDS-PA, however, the time discrepancy between the two models is rather small, which is within the range of 2-11 sampling times difference (see DTD column in Table 3). Meanwhile, the DTD negative values in Table 3 between the two systems is comparatively large from -238 until -14, which clearly shows that there was a great timing lagging of cPCA in detecting the fault compared to the proposed system.

Furthermore, the number of slow detection (SD) cases which cPCA obtained based on at least 10 sampling time delayed is relatively higher compared to CMDS-PA. In particular, there were eleven (11) situations (referring to the '/ marks in Table 3) where cPCA were found sluggish in detection, whereas CMDS-PA merely produced six (6) slow detection cases based on 20 dimensions/PCs models. With respect to the 40 dimensions/PCs models, however, the SD cases for both methods were increased to twelve (12) and eight (8) respectively. This shows that increasing the number of dimensions/PCs will complicate the detection operation rather than simplified. This could be due to the transformation of large variable scores in deducing the monitoring parameters at higher number of dimensions is truly a complex process. Hence, ideal number of dimensions which captured around $70 \%-80 \%$ variance or configuration similarity is highly encouraged.

The most intriguing part of the findings is cPCA suffers from four (4) major NED cases, which consists of abnormal event numbers 3, 9, 15 and 19, whereby no effective detection can be clearly observed on the control chart based on both PCs settings. The NED cases are situations where the signals cannot be maintained at the upper region of the control limits once the fault signal has been injected in the process. 
Table 3. The Summary of FDDT Results for cPCA and CMDS-PA Systems based on 20 and 40 Dimensions

\begin{tabular}{|c|c|c|c|c|c|c|c|c|c|c|}
\hline \multirow[b]{2}{*}{ Fault Cases } & \multicolumn{5}{|c|}{ Dimensions/PCs 20} & \multicolumn{5}{|c|}{ Dimensions/PCs 40} \\
\hline & cPCA & $\begin{array}{c}\text { CMDS- } \\
\text { PA }\end{array}$ & DTD & $\begin{array}{c}\text { cPCA } \\
\text { delayed } \\
\geq 10 \text { st }\end{array}$ & $\begin{array}{c}\text { CMDS- } \\
\text { PA } \\
\text { delayed } \\
\geq 10 \text { st }\end{array}$ & cPCA & $\begin{array}{c}\text { CMDS- } \\
\text { PA }\end{array}$ & DTD & \begin{tabular}{|c|} 
cPCA \\
delayed \\
$>10$ \\
sampling \\
time \\
\end{tabular} & $\begin{array}{l}\text { CMDS- } \\
\text { PA } \\
\text { delayed } \\
\geq 10 \text { st }\end{array}$ \\
\hline 1 & 3 & 7 & 4 & $\mathrm{X}$ & $\mathrm{X}$ & 1 & 7 & 6 & $\mathrm{X}$ & $\mathrm{X}$ \\
\hline 2 & 11 & 15 & 4 & $\mathrm{X}$ & 1 & 5 & 15 & 10 & $X$ & 1 \\
\hline 3 & NED & 1 & - & 1 & $\mathrm{X}$ & NED & 1 & - & 1 & $\mathrm{X}$ \\
\hline 4 & 2 & 13 & 11 & $\mathrm{X}$ & 1 & 2 & 13 & 11 & $\mathrm{X}$ & 1 \\
\hline 5 & 1 & 4 & 3 & $\mathrm{X}$ & $\mathrm{X}$ & 2 & 4 & 2 & $\mathrm{X}$ & $\mathrm{X}$ \\
\hline 6 & 1 & 3 & 2 & $\mathrm{X}$ & $X$ & 3 & 3 & 0 & $\mathrm{X}$ & $X$ \\
\hline 7 & 1 & 1 & 0 & $\mathrm{X}$ & $\mathrm{X}$ & 1 & 1 & 0 & $\mathrm{X}$ & $\mathrm{X}$ \\
\hline 8 & 15 & 1 & -14 & 1 & $\mathrm{X}$ & 17 & 1 & -16 & 1 & $\mathrm{X}$ \\
\hline 9 & NED & 1 & - & 1 & $\mathrm{X}$ & NED & 1 & - & 1 & $\mathrm{X}$ \\
\hline 10 & 40 & 15 & -25 & 1 & 1 & 29 & 15 & -14 & 1 & 1 \\
\hline 11 & 6 & 7 & 1 & $\mathrm{X}$ & $\mathrm{X}$ & 6 & 7 & 1 & $\mathrm{X}$ & $\mathrm{X}$ \\
\hline 12 & 1 & 1 & 0 & $\mathrm{X}$ & $\mathrm{X}$ & 23 & 1 & -22 & 1 & $\mathrm{X}$ \\
\hline 13 & 34 & 1 & -33 & 1 & $\mathrm{X}$ & 37 & 10 & -27 & 1 & $X$ \\
\hline 14 & 1 & 1 & 0 & $\mathrm{X}$ & $\mathrm{X}$ & 1 & 1 & 0 & $\mathrm{X}$ & $\mathrm{X}$ \\
\hline 15 & NED & 95 & - & 1 & 1 & NED & 93 & - & I & 1 \\
\hline 16 & 239 & 1 & -238 & 1 & $\mathrm{X}$ & 11 & 1 & -10 & I & $\mathrm{X}$ \\
\hline 17 & 24 & 31 & 7 & $\mathrm{X}$ & $\mathrm{X}$ & 18 & 31 & 13 & $\mathrm{X}$ & $\mathrm{X}$ \\
\hline 18 & 79 & 25 & -54 & 1 & 1 & 84 & 25 & -59 & I & 1 \\
\hline 19 & NED & 1 & - & 1 & $\mathrm{X}$ & NED & 11 & - & 1 & $\mathrm{X}$ \\
\hline 20 & 81 & 80 & -1 & $\mathrm{X}$ & 1 & 72 & 81 & 9 & $\mathrm{X}$ & 1 \\
\hline NFD & 10 & 13 & & & & 10 & 13 & & & \\
\hline NFD Strength, \% & $50 \%$ & $65 \%$ & & - & & $50 \%$ & $65 \%$ & & - & \\
\hline
\end{tabular}

Legends: NFD: Number of fastest detection cases; '/': Correct; 'X': Incorrect; NED:

No effective detection; DTD: Delayed detection time (DTD=detection time CMDS-PA-detection time cPCA)

For instance, Figure 2 shows the chart progression of $T^{2}$ and SPE of the cPCA system for abnormal cases number 3 , 13 and 15 that operated by using 20 PCs model. From Figure 2, with regard to cases 3 and 15 specifically, both of the events cannot be detected effectively by cPCA via $T^{2}$ or SPE, and as a result, the monitoring outcomes appeared as 'NED' for both cases as denoted in Table 3 previously. On contrary, the fault of case 13 can be detected very effectively by cPCA through both statistics, whereby majority of the samples are located outside the boundaries particularly after the sampling time of 160 (from Table 3 the first detection was notified at sampling time 194 and 197 based on 20 and 40 PCs models respectively).

Figure 3 on the other hand, illustrates the progression of CMDS-PA systems for cases 3, 13 and 15 particularly utilizing dimension 20 model. In particular, Figure 3 shows that the CMDS-PA system has effectively detected cases 3 and 15 comparatively better than that of cPCA, where a large portion of samples are located consistently outside the boundaries after sampling 160. In the case of fault number 13, the CMDS-PA system was also found efficient in detecting the fault much earlier against cPCA as well as a great number of samples are plotted beyond the $99 \%$ limit constantly.

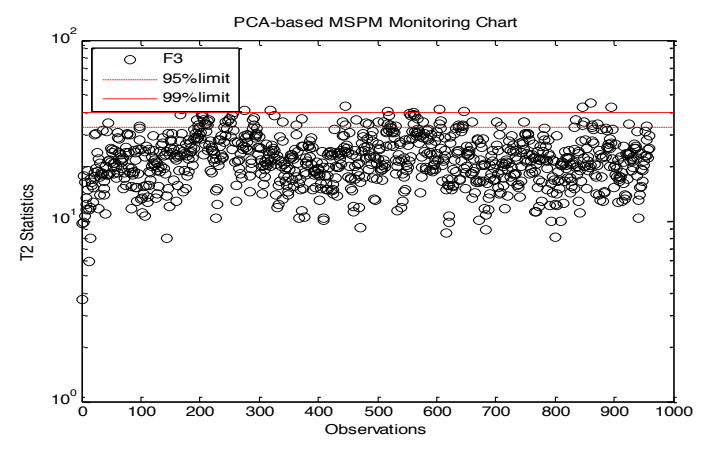

(a)

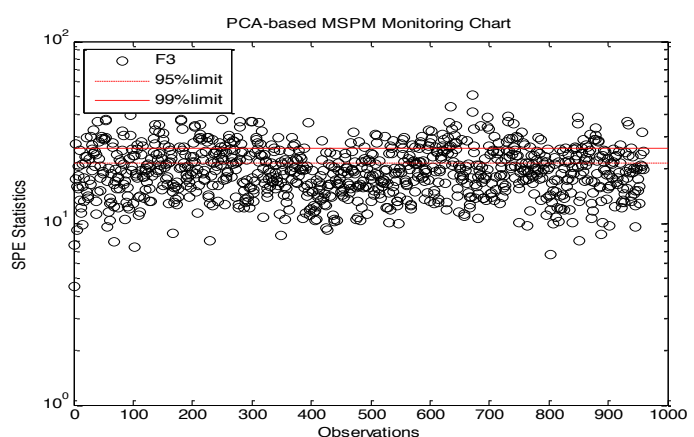

(b) 


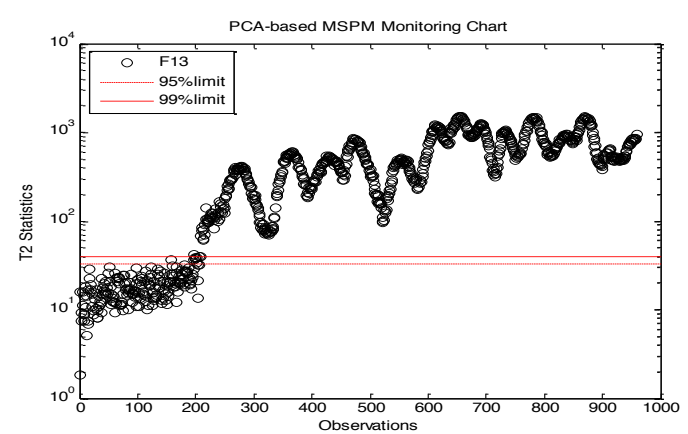

(c)

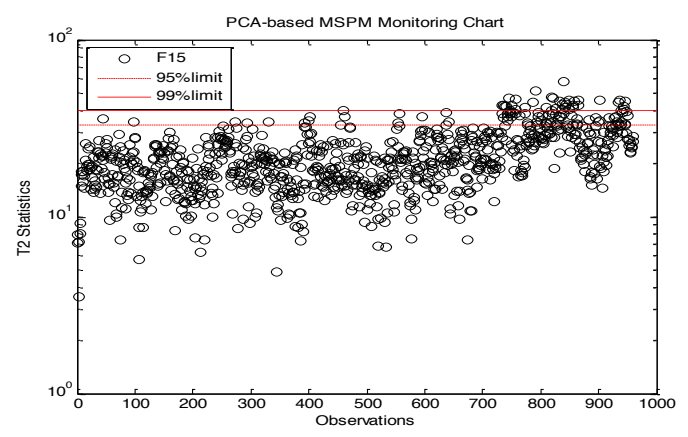

(e)

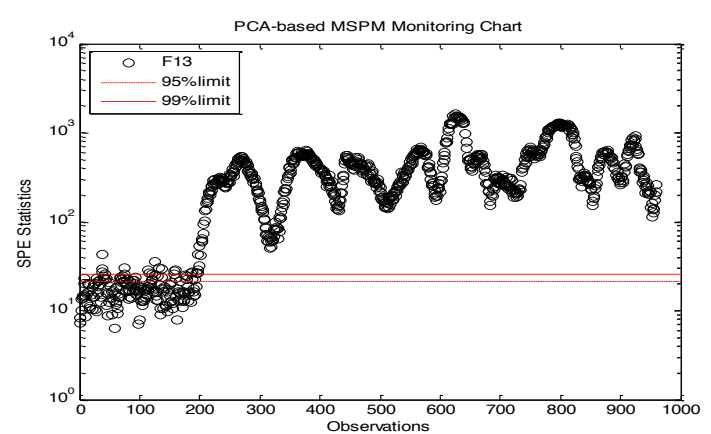

(d)

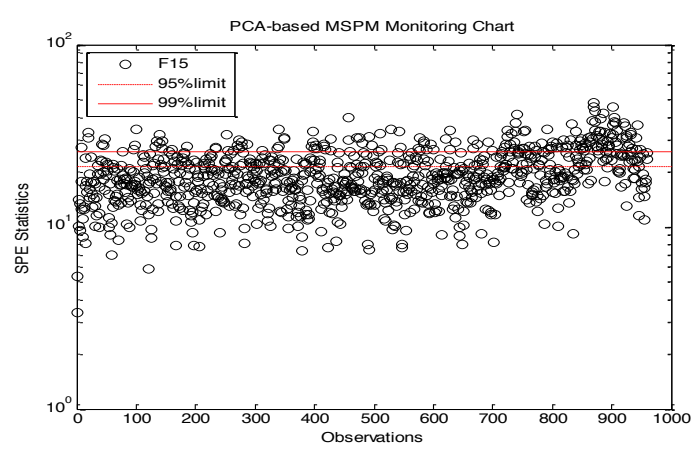

(f)

Figure 2. a) $T^{2}$ Progression of Case 3, b) SPE Progression of Case 3, c) $T^{2}$ Progression of Case 13, d) SPE Progression of Case 13, e) $T^{2}$ Progression of Case 15, f) SPE Progression of Case 15

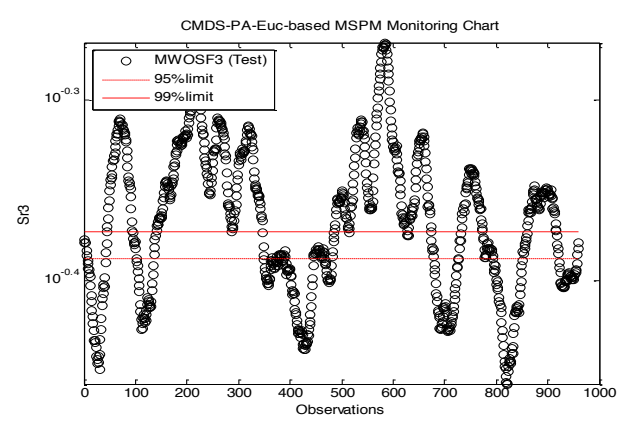

(a)

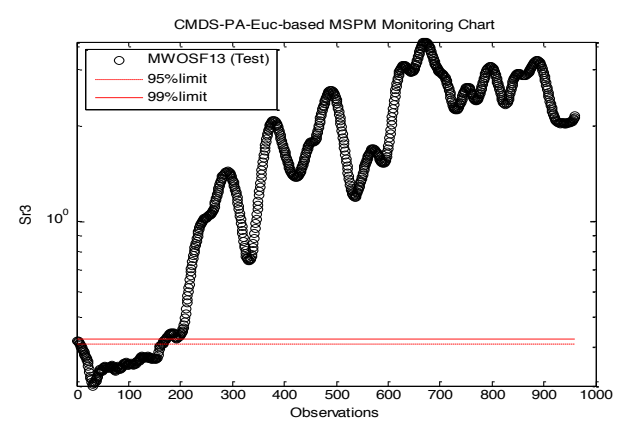

(b)

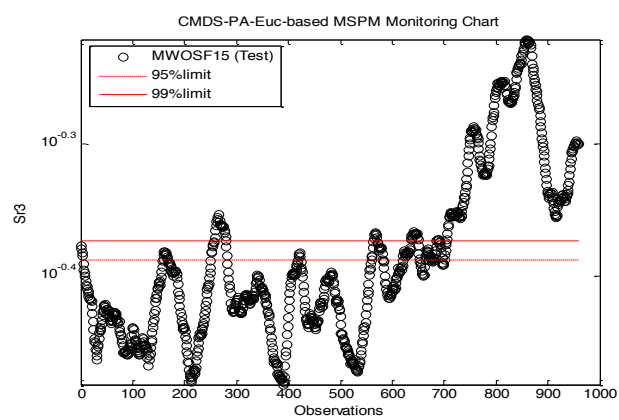

(c)

Figure 3. a) $\mathrm{Sr}_{3}$-CMDS-PA Progression of Case 3, b) $\mathrm{Sr}_{3}$-CMDS-PA Progression of Case 13, c) $\mathrm{Sr}_{3}$-CMDS-PA Progression of Case 15 


\section{CONCLUSION}

This study investigates the application of CMDS-PA monitoring framework particularly on detecting process abnormality based on TEP cases. The study involves the original methodology that discussed in (Yunus, 2012) but with an introduction of a new monitoring statistic, Sr3. This study analysed the performance of the proposed system against the cPCA detection outcomes. It turns out that the CMDS-PA has successfully detected all the faults effectively in relative to $\mathrm{CPCA}$, whereby cPCA was observed struggling in sustaining the signal detection particularly for fault cases numbers 3,9 , and 15. Perhaps, this outstanding performance can be further utilised as well as expanded for the current use of feature monitoring within the realm of big data environment for smart manufacturing (He et al., 2019; He \& Wang, 2018).

\section{ACKNOWLEDGMENT}

The author gratitude goes to the process systems engineering and safety (PROSES) research cluster of FTKKP UMP for their advice and support on this project.

\section{REFERENCES}

Amigo, J. M., Surribas, A., Coello, J., Montesinos, J. L., Maspoch, S., \& Valero, F. (2008). On-line parallel factor analysis. A step forward in the monitoring of bioprocesses in real time. Chemometrics and Intelligent Laboratory Systems, 92(1), 44-52. https://doi.org/10.1016/j.chemolab.2007.12.001

Bakshi, B. R. (1998). Multiscale PCA with application to multivariate statistical process monitoring. AIChE Journal. https://doi.org/10.1002/aic.690440712

Borg, I., \& Groenen, P. (1997). Modern Multidimensional Scaling: Theory and Applications. In Journal of the American Statistical Association (Vol. 94, Issue 445). Springler-Verlag.

Chiang, L. H., Russell, E. L., \& Braatz, R. D. (2000). Fault diagnosis in chemical processes using Fisher discriminant analysis, discriminant partial least squares, and principal component analysis. Chemometrics and Intelligent Laboratory Systems, 50(2), 243-252. https://doi.org/10.1016/S0169-7439(99)00061-1

Cox, T. F. (2003). Multidimensional Scaling in Process Control. In Handbook of Statistics (22nd ed., pp. 609-623). Elsevier Science B.V.

Cox, T. F. (2005). An Introduction to Multivariate Data Analysis. Hodder Education.

Cox, T. F., \& Cox, M. A. A. (1994). Multidimensional Scaling. Chapman \& Hall.

Cox, Trevor F. (2001). Multidimensional scaling used in multivariate statistical process control. Journal of Applied Statistics, 28(3-4), 365-378. https://doi.org/10.1080/02664760120034108

Downs, J. J., \& Vogel, E. F. (1993). A plant-wide industrial process control problem. Computers and Chemical Engineering, 17(3), 245-255. https://doi.org/10.1016/0098-1354(93)80018-I

Everitt, B. S., \& Jackson, J. E. (1992). A User's Guide to Principal Components. In Biometrics (Vol. 48, Issue 3). https://doi.org/10.2307/2532367

He, Q. P., \& Wang, J. (2018). Statistical process monitoring as a big data analytics tool for smart manufacturing. Journal of Process Control, 67, 35-43. https://doi.org/10.1016/j.jprocont.2017.06.012

He, Q. P., Wang, J., \& Shah, D. (2019). Feature space monitoring for smart manufacturing via statistics pattern analysis. Computers and Chemical Engineering, 126, 321-331. https://doi.org/10.1016/j.compchemeng.2019.04.010

Ibrahim, K. A. (1997). Application of Partial Correlation Analysis in Active Statistical Process Control. Proceedings of Regional Symposium of Chemical Engineering, 434 - 439.

Juricek, B. C., Seborg, D. E., \& Larimore, W. E. (2000). Identification of the Tennessee Eastman Challenge Process with Subspace Methods. IFAC Proceedings Volumes, 33(15), 409-414. https://doi.org/10.1016/s1474-6670(17)397859

Kourti, T., \& MacGregor, J. F. (1995). Process analysis, monitoring and diagnosis, using multivariate projection methods. In Chemometrics and Intelligent Laboratory Systems. https://doi.org/10.1016/0169-7439(95)80036-9

Kruskal, J.B., and Wish, M. (1978). Multidimensional scaling. SAGE Publications.

$\mathrm{Ku}$, W., Storer, R. H., \& Georgakis, C. (1995). Disturbance detection and isolation by dynamic principal component analysis. Chemometrics and Intelligent Laboratory Systems, 30(1), 179-196. https://doi.org/10.1016/01697439(95)00076-3

Lee, J. M., Yoo, C. K., \& Lee, I. B. (2004). Statistical process monitoring with independent component analysis. Journal of Process Control. https://doi.org/10.1016/j.jprocont.2003.09.004

MacGregor, J. F., \& Kourti, T. (1995). Statistical process control of multivariate processes. Control Engineering Practice. https://doi.org/10.1016/0967-0661(95)00014-L

MacGregor, John F., Jaeckle, C., Kiparissides, C., \& Koutoudi, M. (1994). Process monitoring and diagnosis by multiblock PLS methods. AIChE Journal. https://doi.org/10.1002/aic.690400509

Martin, E. B., Morris, A. J., \& Zhang, J. (1996). Process performance monitoring using multivariate statistical process control. IEE Proceedings: Control Theory and Applications. https://doi.org/10.1049/ip-cta:19960321

Molina, G. D., Zumoffen, D. A. R., \& Basualdo, M. S. (2011). Plant-wide control strategy applied to the Tennessee Eastman process at two operating points. Computers and Chemical Engineering, 35(10), 2081-2097. https://doi.org/10.1016/j.compchemeng.2010.11.006

Nomikos, P., \& MacGregor, J. F. (1995). Multivariate SPC charts for monitoring batch processes. Technometrics. 
https://doi.org/10.1080/00401706.1995.10485888

Oppenheim, A. N., \& Torgerson, W. (1961). Theory and Methods of Scaling. In The British Journal of Sociology (Vol. 12, Issue 1). John Wiley \& Sons. https://doi.org/10.2307/588039

Takane, Y. (2004). Matrices with special reference to applications in psychometrics. Linear Algebra and Its Applications, 388(1-3 SPEC. ISS.), 341-361. https://doi.org/10.1016/S0024-3795(03)00451-8

Yunus, M. Y. B. M. (2012). Multivariate Statistical process Monitoring using Classical Multidimensional Scaling. Newcastle University.

Zhang, J., Martin, E. B., \& Morris, A. J. (1997). Process monitoring using non-linear statistical techniques. Chemical Engineering Journal. https://doi.org/10.1016/S1385-8947(97)00048-X

Zheng, A. (1998). Non-linear Model Predictive Control of the Tennessee Eastman Process. Proceeding of the American Control Conference. 